\title{
The Fibrolytic Enzyme Profiles and the Composition of Fungal Communities in Donkey Cecum-Colon Ecosystem
}

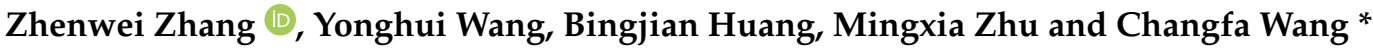

check for updates

Citation: Zhang, Z.; Wang, Y.; Huang, B.; Zhu, M.; Wang, C. The Fibrolytic Enzyme Profiles and the Composition of Fungal Communities in Donkey Cecum-Colon Ecosystem. Animals 2022, 12, 412. https:// doi.org/10.3390/ani12040412

Academic Editor: Chris W. Rogers

Received: 16 January 2022

Accepted: 8 February 2022

Published: 9 February 2022

Publisher's Note: MDPI stays neutral with regard to jurisdictional claims in published maps and institutional affiliations.

Copyright: (C) 2022 by the authors. Licensee MDPI, Basel, Switzerland. This article is an open access article distributed under the terms and conditions of the Creative Commons Attribution (CC BY) license (https:// creativecommons.org/licenses/by/ $4.0 /)$.
Liaocheng Research Institute of Donkey High-Efficiency Breeding and Ecological Feeding, Liaocheng University, Liaocheng 252059, China; qingyibushuo@163.com (Z.Z.); wyh19920729@163.com (Y.W.); 17853148163@163.com (B.H.); zhumingxia@lcu.edu.cn (M.Z.)

* Correspondence: wangcf1967@163.com

\begin{abstract}
Simple Summary: The donkey hindgut is a microbial-rich ecosystem in which caecum and colon fungi play an important role in dietary fiber degradation. In addition, the fibrolytic enzymes produced by hindgut microorganisms are key to the ability of equines to hydrolysis plant fiber. In the present study, the fibrolytic enzyme activities within donkey caecum and colon were firstly measured by spectrophotometry. The dorsal colon presented a higher fibrolytic enzyme activity in comparison with caecum. The fungal community composition along donkey caecum and colon was determined by sequencing an internal transcribed spacer region (ITS) using Illumina MiSeq. The predominant fungi at phylum level were Ascomycota, Basidiomycota, and Neocallimastigomycota. The Aspergillus, Wallemia, Phanerochaete, Fusarium, and Penicillium were detected as the dominant genera, but their metabolic and functional significance in donkey cecum-colon ecosystem need further investigation. In terms of the anaerobic fungi Neocallimastigomycota, its abundance was greater in donkey colon than in caecum. The relative abundance of enzymes related to plant cell wall breakdown were also predicted by PICRUSt, and they were also greater in donkey colon than in caecum. The present study provided new information about fibrolytic enzyme profiles and fungal communities in donkey hindgut. The findings could therefore contribute to the further understanding of the fungal taxa and their dietary fiber degradation mechanisms in donkey hindgut ecosystem.
\end{abstract}

Abstract: The fibrolytic enzymes and the hindgut fungi in donkey cecum-colon ecosystem play an important role in dietary fiber digestion. A better understanding of the fibrolytic enzyme profiles and the fungal community along donkey caecum and colon is key for optimizing hindgut function. In the present study, the fibrolytic enzyme activities within donkey caecum and colon were firstly measured by spectrophotometry. Activities of carboxymethyl cellulase, avicelase, xylanase, and acetyl esterase were greater in donkey dorsal colon than in caecum, indicating that the colon microorganisms may be more efficient in producing fibrolytic enzymes compared to caecum microbes. The fungal community composition along donkey hindgut was determined by sequencing ITS region using Illumina MiSeq. Three fungal phyla were identified by sequence comparison: Ascomycota $(66.8 \%-74.4 \%)$, Basidiomycota $(21.6 \%-30.9 \%)$, and Neocallimastigomycota (0.9\%-3.3\%). The Aspergillus, Wallemia, Phanerochaete, Fusarium, and Penicillium were detected as the dominant genera, but their metabolic and functional significance in donkey cecum-colon ecosystem need further investigation. In terms of the anaerobic fungi Neocallimastigomycota, its abundance was greater in donkey colon than in caecum $(p<0.05)$, indicating that the donkey hindgut region was associated with differences in fungal community composition. Moreover, the relative abundance of enzymes related to plant cell wall degradation were predicted by PICRUSt, and they were also lower in caecum than in colon. The present study provided new information about fibrolytic enzyme profiles and fungal composition in donkey hindgut ecosystem.

Keywords: donkey; caecum; colon; fibrolytic enzyme; fungal composition 


\section{Introduction}

Donkeys have evolved as free-grazing herbivores with a specialized and enlarged hindgut. The equine hindgut is mainly comprised of two fermentative compartments, the caecum and colon, which together account for two-thirds of the volume of donkey's gastrointestinal tract [1]. Microbial fermentation of plant structural polysaccharides in donkey hindgut results in the production of volatile fatty acids (VFAs), which are a major source of energy for the host [2].

Among hindgut microorganisms, both the bacteria and the fungi play a pivotal role in the hindgut fermentation and the degradation of feedstuffs [2]. Nowadays, increasing studies have been successfully performed to reveal the changes and functions of the hindgut bacteria community in equines [3,4]. However, little information is available regarding the composition of fungal communities in the equine hindgut ecosystem. The current knowledge of gut fungi has focused on the strictly anaerobic fungi (phylum Neocallimastigomycota) and is mostly derived from ruminant based studies $[5,6]$.

Based on physiological and developmental characteristics, Neocallimastigomycota is generally divided into the monocentric rhizoidal, the polycentric rhizoidal, and the bulbous genera [5]. Anaerobic fungi were reported to be present in domesticated and nondomesticated equine species, and their diversity in donkeys was shown to be higher when compared to that of ponies and pony $\times$ donkey hybrids [2]. Recently, Edwards et al. determined the fecal community composition of anaerobic fungi in donkeys, and the genus Caecomyces, SK3, KF1, and Piromyces were found in the fecal samples [2]. The presence of these anaerobic fungi may enable the donkeys to utilize fibrous feeds efficiently and persistently. However, recent studies in ruminant animals have shown that Ascomycota and Basidiomycota phyla also play an important role in the rumen digestion of cashmere goats and Holstein cows $[7,8]$.

The fungal ribosomal RNA (rRNA) operon-based analysis has facilitated the understanding of the diversity and phylogenetic relationships of gut fungi in natural ecosystems [9]. The internal transcribed spacer (ITS) region is usually recommended as the DNA barcode marker for gut fungi $[10,11]$. In addition, the $28 \mathrm{~S}$ ribosomal large subunit (28S rRNA gene (LSU); D1/D2 region) might render higher coverage of early diverging lineages of fungi, such as Neocallimastigomycota, Chytridiomycota, and Mucoromycota [11]. However, there is still a big challenge of how to relate LSU taxonomic sequences to the clades that until now only contained "unculturable representatives" derived ITS1 sequences [5,12]. Therefore, the ITS region remains the most accepted phylogenetic marker to assess the diversity and community structure of gut fungi.

Due to ethical noninvasive sampling, fecal samples are commonly used for fungi investigation in equines [2,7]. The understanding of equine fungi diversity and community structure within different hindgut regions is limited. It is very necessary to evaluate the diversity and composition of hindgut fungi in the anatomically specialized hindgut of donkeys. Moreover, both the bacteria and the fungi produce a broad array of fibrolytic enzymes that can facilitate the plant fragments degradation by breaking the linkages between lignin and hemicelluloses $[13,14]$. The digestibility of plant fiber in donkeys may be to a large extent dependent on the strong fibrolytic activity of the main-chain or the side-chain degrading enzymes, including carboxymethyl cellulase, xylanase, ferulic acid esterase, and acetyl esterase. However, neither the main-chain degrading enzymes nor the side-chain degrading enzymes have been reported in the donkey caecum and colon.

Therefore, the fibrolytic enzyme profiles were firstly determined to assess functional differences in digesting plant cell wall within the different hindgut regions in donkeys. Furthermore, the primary objective of the present study is to comparatively analyze the fungal community composition along the hindgut segments (caecum and colon) of donkeys using Illumina MiSeq sequencing by covering the ITS region. 


\section{Materials and Methods}

\subsection{Ethics Statement}

The animal care and sample procedures were approved by the Institutional Animal Care Committee at Liaocheng University (Permit No. DFG21010103-1).

\subsection{Animals and Diets}

Eight jackasses (weighing $303 \pm 18 \mathrm{~kg}$, 2.5 years of age) belonging to the Dezhou donkey breed were enrolled. They were reared in a large-scale donkey farm in northern China and housed in stalls. Donkeys were fed twice daily at 07:00 and 19:00 h. Diet was based on corn straw ad libitum along with a commercial concentrate feed, according to the nutrient requirements reported by the NRC recommendations [15]. Donkeys had free access to water at all times. In addition, donkeys were healthy, with no history of any intestinal disorders.

\subsection{Sample Collection}

Donkeys were euthanized for non-research reasons in a local abattoir. After fasting for $12 \mathrm{~h}$, they were knocked unconscious using electronarcosis of $220 \mathrm{~V}$ for $20 \mathrm{~s}$, and then slaughtered by exsanguination using conventional humane procedures. After exteriorization of the gastrointestinal tract, the caecum, ventral colon, and dorsal colon were tied off to avoid mixing between adjacent segments. The hindgut contents from one section were collected immediately with press filtration through four layers of cheesecloth and mixed thoroughly to obtain the representative caecum, ventral, and dorsal colon sample (liquid and small particles). An aliquot of each sample $(2 \mathrm{~mL})$ was transferred to the labelled sterile tube and transported on wet ice back to the laboratory where they were stored at $-70{ }^{\circ} \mathrm{C}$ until DNA extraction. A second aliquot $(10 \mathrm{~mL})$ of each sample was transferred to polypropylene tube and centrifuged at $1000 \times \mathrm{g}$ at $4{ }^{\circ} \mathrm{C}$ for $15 \mathrm{~min}$, and the supernatant was immediately analyzed for fibrolytic enzyme profile analysis.

\subsection{Fibrolytic Enzyme Profile Measurement}

The activity of cellulolytic enzyme including carboxymethyl cellulose (CMCase), avicelase (AVI), xylanase (XYL), feruloyl esterase (FAE), and acetyl esterase (AE) were measured by the spectrophotometer (Bio-rad, Hercules, CA, USA), as described previously [16]. For FAE activity determination, the primary centrifugal supernatants from caecum, ventral colon, and dorsal colon fluids were incubated with 2 vol of $100 \mu \mathrm{M}$ methyl ferulate in $100 \mathrm{mM}$ 3-(N-morpholino) propane sulfonic buffer at $39^{\circ} \mathrm{C}$ for $30 \mathrm{~min}$, and the absorbance was detected by spectrophotometer at $340 \mathrm{~nm}$. Enzyme activity was calculated according to standard curves of feruloyl acid (FA) and methyl ferulate (MA):

Activity $(\mathrm{U})=\left[\left(\mathrm{OD}_{0}-\mathrm{OD}_{30}\right)-\left(\mathrm{OD}_{\mathrm{b} 0}-\mathrm{OD}_{\mathrm{b}}\right)\right] \times \mathrm{V}_{\mathrm{r}} \times$ Dilution $/\left[(\varepsilon \mathrm{MA} \times l-\varepsilon \mathrm{FA} \times l) \times \mathrm{V}_{\mathrm{s}}\right]$

where $\mathrm{OD}_{0}$ and $\mathrm{OD}_{30}$ is the absorbance of reaction system at 0 and $30 \mathrm{~min}$, respectively; $\mathrm{OD}_{\mathrm{b} 0}$ and $\mathrm{OD}_{\mathrm{b} 30}$ is the absorbance of blank system at 0 and $30 \mathrm{~min}$, respectively; $\mathrm{V}_{\mathrm{r}}$ and $\mathrm{V}_{\mathrm{s}}$. is the volume of reaction system and sample, respectively; $\varepsilon \mathrm{MA}$ is the extinction coefficient of methyl ferulate; $\varepsilon F A$ is the extinction coefficient of feruloyl acid; and $l$ is the pathlength.

The activities of AE, CMCase, AVI, and XYL were measured colorimetrically by using p-nitrophenyl acetate, carboxymethyl cellulose, avicael, and xylan as the substrate, respectively. The production of $p$-nitrophenol was determined at $415 \mathrm{~nm}$, and the production of reducing sugars was quantified colorimetrically at $540 \mathrm{~nm}$ by the dinitrosalicylic acid method [17]. One unit of enzyme activity was defined as the amount of enzyme releasing $1.0 \mu \mathrm{mol}$ of corresponding reducing products (feruloyl acid or reducing sugar or $p$-nitrophenol) $/ \mathrm{min} / \mathrm{mL}$ under the aforementioned conditions. 


\subsection{The DNA Extraction and PCR Amplification}

Following the instructions, genomic DNA was extracted from caecum and colon fluids samples using the E.Z.N.A. ${ }^{\circledR}$ soil DNA Kit (Omega Bio-tek, Norcross, GA, USA). After the evaluation of quality and quantity of extracted DNA, four samples from each caecum, ventral colon, and dorsal colon were selected and delivered to Majorbio Company (Shanghai, China) for PCR amplification and MiSeq sequencing.

For anaerobic fungal community composition analysis, barcoded amplicons comprising the ITS1-ITS2 were generated with primer pairs ITS1F ( $5^{\prime}$-CTTGGTCATTTAGAGGAAGTAA$\left.3^{\prime}\right)$ [18] and ITS2R (5'-GCTGCGTTCTTCATCGATGC-3') [19] by an ABI GeneAmp ${ }^{\circledR} 9700$ PCR thermocycler (ABI, Arlington, VA, USA). The PCR amplification of ITS1 ITS2 gene was performed in a total volume of $20 \mu \mathrm{L}$ (Table 1). The PCR were carried out in triplicate and using the appropriative thermal cycling conditions. The PCR product (350 bp) was excised from 2\% agarose gel and purified by the AxyPrep DNA Gel Extraction Kit (Axygen Biosciences, Union City, CA, USA). The DNA concentrations of PCR products were measured using Quantus ${ }^{\mathrm{TM}}$ Fluorome-ter (Promega, Fitchburg, WI, USA) and amplicons from each reaction mixture were pooled at equimolar ratios based on the concentration of each amplicon.

Table 1. The reaction system for PCR amplification.

\begin{tabular}{cc}
\hline Items & Volume \\
\hline $10 \times$ TaKaRa rTaq buffer & $2 \mu \mathrm{L}$ \\
2.5 mM dNTPs & $2 \mu \mathrm{L}$ \\
$5 \mu \mathrm{M}$ forward primer & $0.8 \mu \mathrm{L}$ \\
$5 \mu \mathrm{M}$ reverse primer & $0.8 \mu \mathrm{L}$ \\
TrTaq Polymerase & $0.2 \mu \mathrm{L}$ \\
template DNA & $10 \mathrm{ng}$ \\
ddd $_{2} \mathrm{O}$ & up to $20 \mu \mathrm{L}$ \\
\hline
\end{tabular}

\subsection{The Illumina MiSeq Sequencing}

Following the standard protocols of Majorbio Bio-Pharm Technology Co. Ltd. (Shanghai, China), the purified PCR amplicons were pooled in equimolar and paired-end sequenced $(2 \times 300)$ on an Illumina MiSeq platform (Illumina, San Diego, CA, USA). The raw reads were deposited into the NCBI Sequence Read Archive (SRA) database (PRJNA7797744).

\subsection{Processing of Anaerobic Fungal Sequence Data}

The fungal sequence data was then quality-filtered using Trimmomatic and merged by FLASH v.1.2.11 with the standard protocols described by Wang et al. [20]. After the removal of chimeric sequences, operational taxonomic units (OTUs) with $97 \%$ similarity cutoff were clustered by UPARSE (version 7.1). Venn diagrams were built using the $\mathrm{R}$ language (version 3.3.1). The taxonomy of each OTU representative sequence was identified by Ribosomal Database Project (RDP v.2.11) Classifier against the unite fungi ITS database (version 8.0) using confidence threshold of 0.97 . The coverage and sampling effort were estimated by the determination of Good's coverage and rarefaction curves. Unweighted and weighted uniFrac distance-based principal coordinate analysis (PCoA), fungal diversity (Shannon and Simpson index), and richness (Sobs and Chao index) were assessed by the programMothur (version v.1.30.2). The composition of each sample at each taxonomic level was determined and plotted into taxonomy histograms. The relative abundance of fungal community was expressed as a percentage. Kruskal-Wallis $\mathrm{H}$ test was used to assess the difference in the relative abundance of fungi. Fungal enzymatic abundance was predicted using PICRUSt v.1.1.0 (Phylogenetic Investigation of Communities by Reconstruction of Unobserved States, Paris, 2012, Curtis Huttenhower) software. 


\subsection{Statistical Analysis}

Univariate data (fibrolytic enzyme profiles) were analyzed using the MIXED procedure of SAS 9.4. The MIXED included the fixed effect of donkey hindgut region, and the random and repeated effect of the animal $(n=8)$ as covariate. Least square mean and standard error of means (SEM) were calculated with the LSMEAN procedure of the SAS 9.4. Differences were considered at $p<0.05$, whereas $0.05<p<0.10$ were declared to be a trend. Analyses of fungal community data and ITS predicted functional profiles were carried out using the i-Sanger platform (http:/ / www.i-sanger.com/, accessed on 15 January 2022).

\section{Results}

\subsection{Fibrolytic Enzyme Profiles}

Apart from the FAE, the activities of CMCase, AVI, XLY, and AE in caecum and ventral colon were all lower than that in dorsal colon (Table $2, p<0.05$ ).

Table 2. The fibrolytic enzyme profiles in cecum, ventral colon, and dorsal colon of Dezhou donkeys.

\begin{tabular}{cccccc}
\hline Items & Caecum & Ventral Colon & Dorsal Colon & SEM & $p$ \\
\hline CMCase, $U$ & $0.13^{\mathrm{b}}$ & $0.10^{\mathrm{b}}$ & $0.37^{\mathrm{a}}$ & 0.066 & 0.02 \\
AVI, U & $0.14^{\mathrm{b}}$ & $0.12^{\mathrm{b}}$ & $0.23^{\mathrm{a}}$ & 0.015 & $<0.01$ \\
XLY, U & $2.5^{\mathrm{b}}$ & $2.0^{\mathrm{b}}$ & $7.3^{\mathrm{a}}$ & 0.570 & $<0.01$ \\
FAE, mU & $4.2^{\mathrm{b}}$ & $4.5^{\mathrm{b}}$ & $4.5^{\mathrm{a}}$ & 0.47 & 0.85 \\
AE, mU & $21.6^{\mathrm{b}}$ & $20.2^{\mathrm{b}}$ & $30.2^{\mathrm{a}}$ & 1.72 & $<0.01$ \\
\hline
\end{tabular}

a,b Values in the same row with different small letter superscripts represent significant difference $(p<0.05)$, while with no letter or the same letter superscripts represent no significant difference $(p>0.05)$. CMCase, carboxymethyl cellulase; AVI, avicelase; XLY, xylanase; FAE, ferulic acid esterase; AE, acetyl esterase; SEM, mean standard error.

\subsection{Alpha Diversity}

In the present study, indices of alpha diversity included Shannon, Simpson, Chao, and Sobs index (Figure 1). No difference occurred among donkey caecum, ventral colon, and dorsal colon $(p>0.05)$.

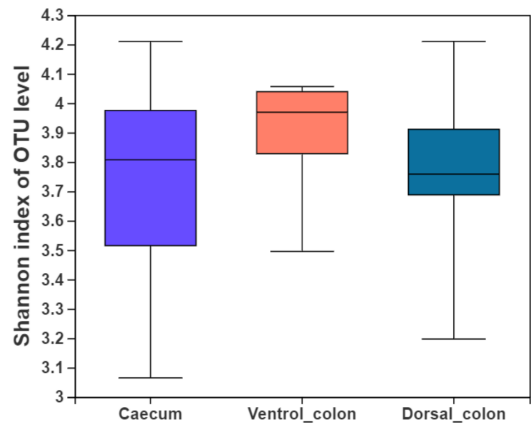

(a)

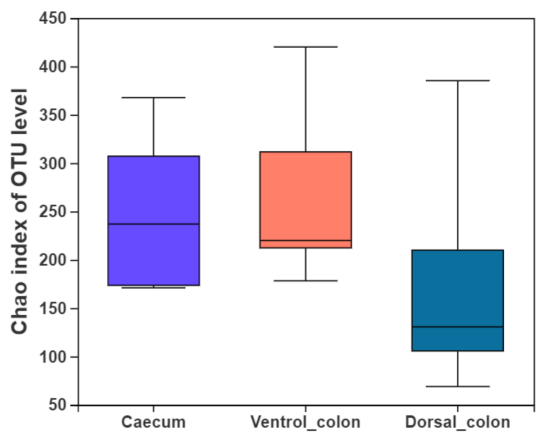

(c)

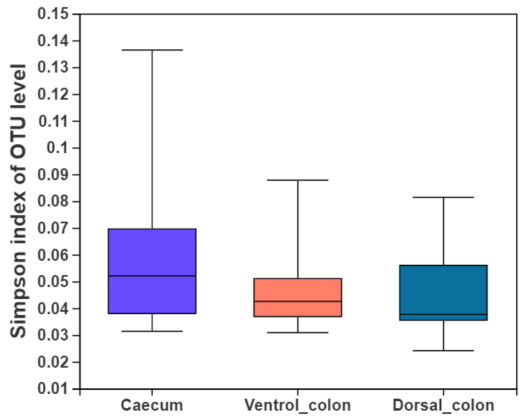

(b)

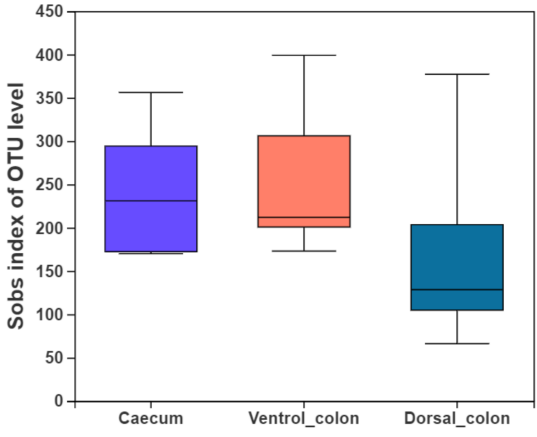

(d)

Figure 1. Fungal alpha diversity indices of caecum and colon in Dezhou donkeys. (a), Shannon index; (b), Simpson index; (c), Chao index; (d), Sobs index. 


\subsection{Fungal Community Composition}

As shown in Figure 2, Venn diagram presented the distribution of fungal community OTUs. There were 702, 711, and 563 OTUs that were observed in donkey caecum, ventral colon, and dorsal colon, respectively. In addition, caecum shares the fungal community, including 343 OTUs, with ventral colon and dorsal colon.

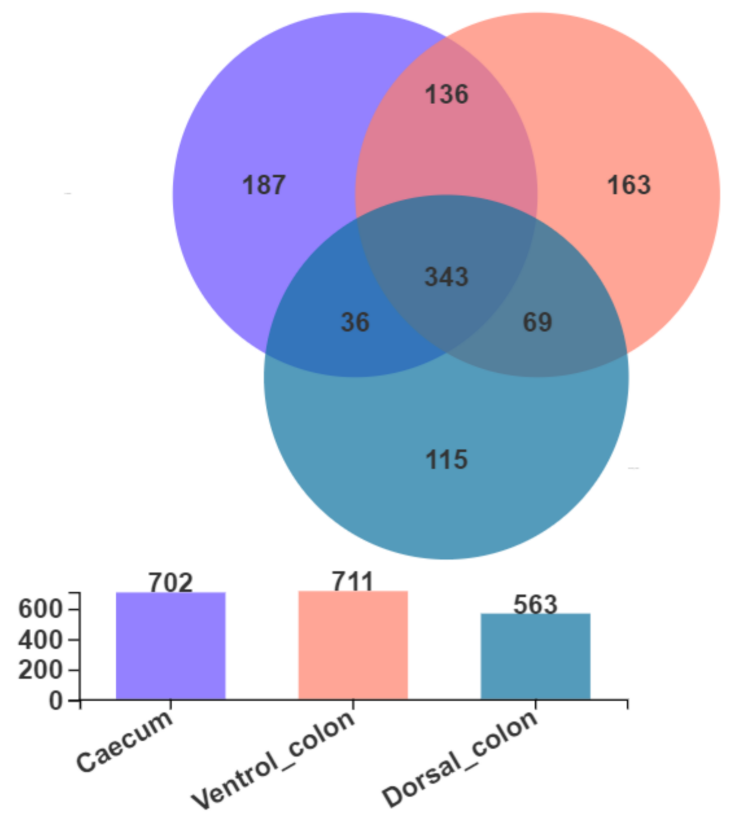

Figure 2. Venn diagram presented the distribution of fungal community OTUs across the caecum, ventral colon, and dorsal colon.

Fungi with a relative abundance of $\geq 1 \%$ of the total sequences in at least one of the samples were further analyzed (Figure 3). The top four predominant phylum were Ascomycota (66.8\%-74.4\% of the total sequence reads), Basidiomycota $(21.6 \%-30.9 \%)$, Neocallimastigomycota $(0.9 \%-3.3 \%)$ and unclassified fungi $(1.0 \%-1.3 \%)$.

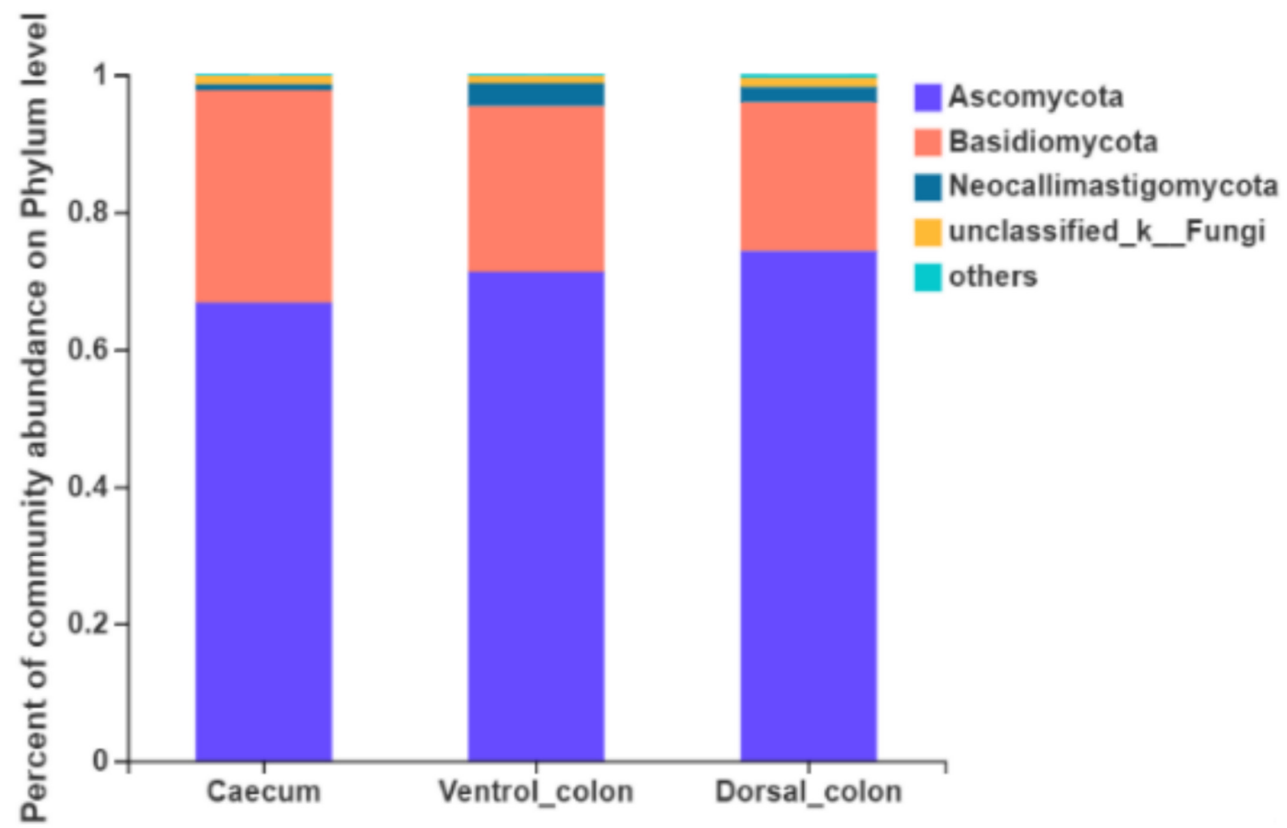

Figure 3. Composition of the predominant fungal phyla (relative abundance $\geq 1 \%$ ) among donkey caecum, ventral colon, and dorsal colon $(n=8)$. 
The relative abundance of Neocallimastigomycota in donkey caecum was significantly lower than that in the ventral and dorsal colon (Figure $4 ; p=0.03$ ).

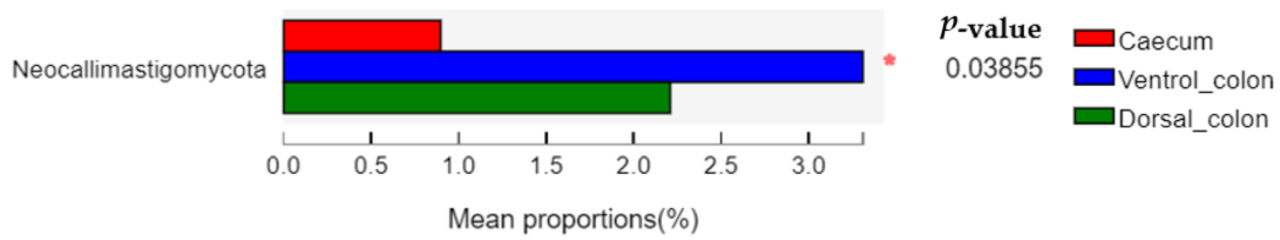

Figure 4. Difference in the relative abundance of fungal phyla (abundance of the phyla was expressed as \%). Welch's two-sided test was used and Welch's inverted was 0.95. ${ }^{*}, p<0.05$.

At the genus level, the top 10 predominant genera were Aspergillus, Wallemia, Phanerochaete, Fusarium, Candida, unclassified_Dipodascace, Talaromyces, Thermomyces, Acremonlum, and Pithoascus (Figure 5).

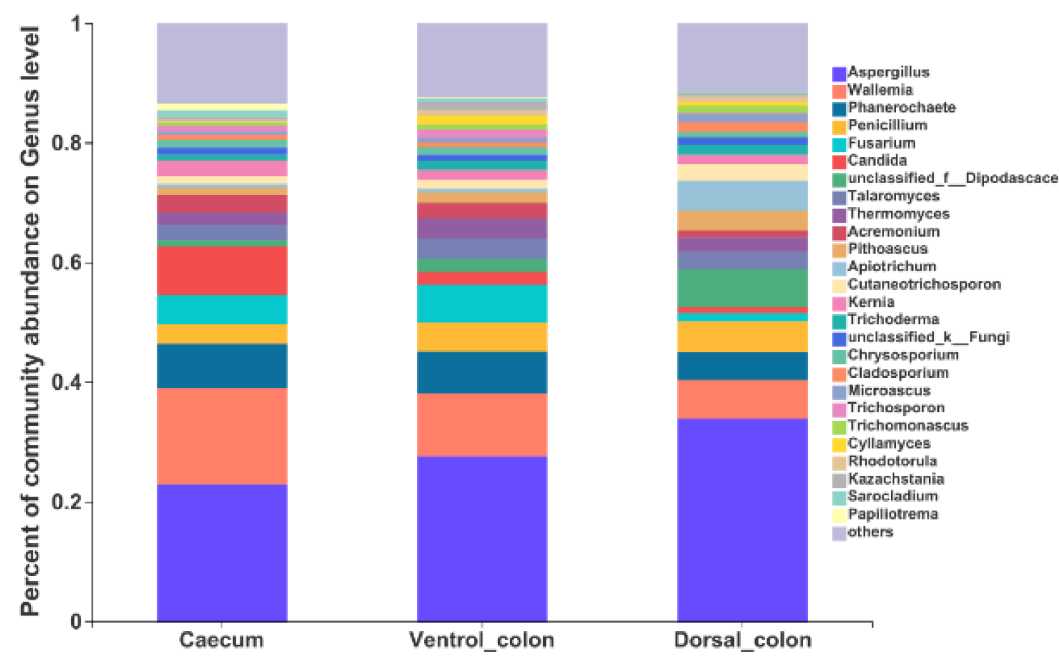

Figure 5. Composition of the fungal genera (relative abundance $>1 \%$ ) among donkey caecum, ventral colon, and dorsal colon $(n=8)$.

The relative abundance of Fusarium and Trichosporon in donkey hindgut was ranked as: ventral colon $>$ caecum $>$ dorsal colon (Figure 6; $p<0.05$ ). In addition, the relative abundance of unclassified Dipodascaceae and Cyllamyces in donkey colon was significantly greater than that in the caecum $(p<0.05)$.

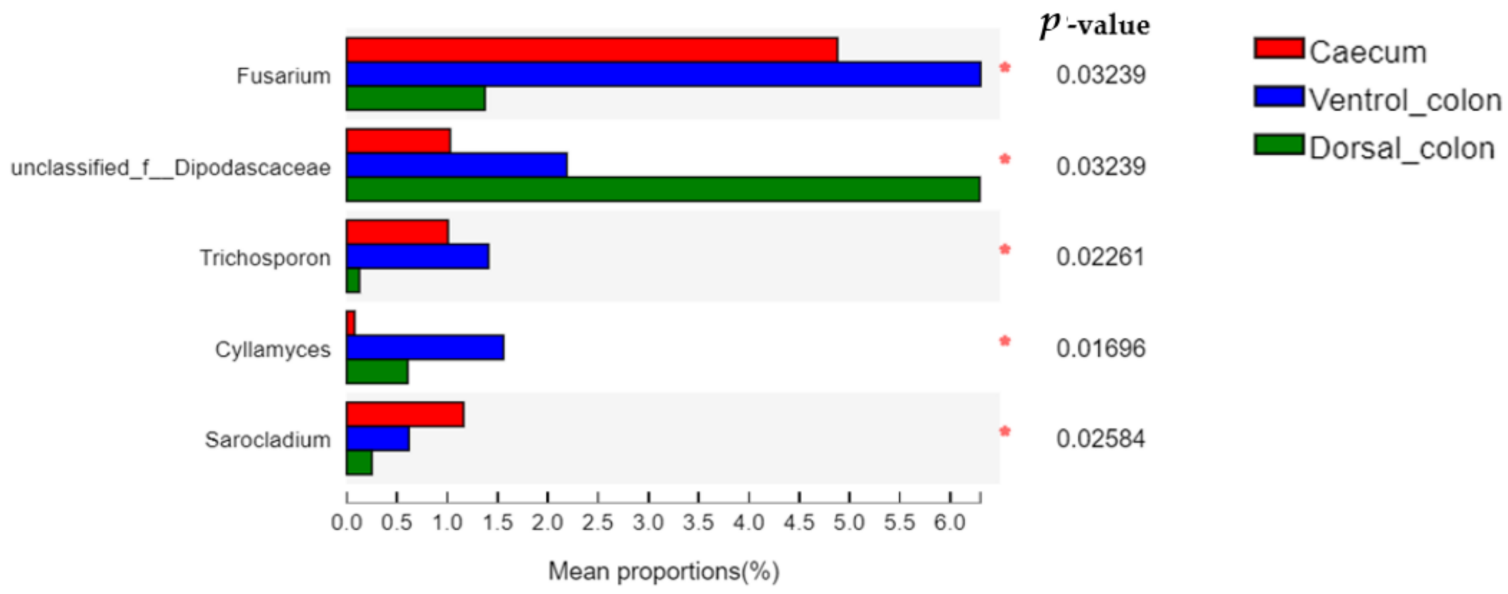

Figure 6. Difference in the relative abundance of fungal genera (abundance of the phyla was expressed as \%). Welch's two-sided test was used and Welch's inverted was $0.95 .^{*}, p<0.05$. 
The anaerobic fungal community belonging to the Neocallimastigomycota phylum were further analyzed (Table 3). The genera Cyllamyces, Neocallimastix, Piromyces, Buwchfawromyces, Unclassified_f_Neocallimastigaceae, and unclassified_c_Neocallimastigomycetes were detected within donkey hindgut. The abundance of anaerobic fungal community composition varied greatly between donkey individuals. In addition, the relative abundance of anaerobic fungi was numerically lower in the caecum than in both ventral colon and dorsal colon.

Table 3. The relative abundance of anaerobic fungal genera composition (belonging to the Neocallimastigomycota phylum) in donkey caecum and colon.

\begin{tabular}{|c|c|c|c|c|}
\hline Items & & Caecum & Ventral Colon & Dorsal Colon \\
\hline \multirow{3}{*}{ Cyllamyces } & Min & 0.008 & 0.011 & 0.002 \\
\hline & Max & 0.589 & 3.81 & 2.16 \\
\hline & Mean \pm SD & $0.21 \pm 0.19$ & $0.71 \pm 0.52$ & $0.69 \pm 0.32$ \\
\hline \multirow{3}{*}{ Piromyces } & Min & 0.002 & 0.002 & 0.009 \\
\hline & Max & 0.346 & 1.21 & 3.34 \\
\hline & Mean \pm SD & $0.12 \pm 0.11$ & $0.42 \pm 0.18$ & $1.24 \pm 1.05$ \\
\hline \multirow{3}{*}{ Neocallimastix } & Min & ND & 0.003 & ND \\
\hline & Max & 0.002 & 2.67 & 5.66 \\
\hline & Mean \pm SD & 0.002 & $0.68 \pm 0.66$ & 5.66 \\
\hline \multirow{3}{*}{ Buwchfawromyces } & Min & 0.006 & 0.003 & ND \\
\hline & Max & 0.014 & 1.88 & 0.95 \\
\hline & Mean \pm SD & $0.01 \pm 0.004$ & $0.94 \pm 0.93$ & 0.95 \\
\hline \multirow{3}{*}{ Unclassified_f_Neocallimastigaceae } & Min & 0.002 & 0.013 & 0.049 \\
\hline & Max & 0.066 & 4.78 & 0.838 \\
\hline & Mean $\pm S D$ & $0.03 \pm 0.02$ & $0.83 \pm 0.57$ & $0.41 \pm 0.13$ \\
\hline \multirow{3}{*}{ Unclassified_c_Neocallimastigomycetes } & Min & ND & 0.002 & 0.003 \\
\hline & Max & ND & 0.18 & 0.03 \\
\hline & Mean $\pm S D$ & ND & $0.06 \pm 0.04$ & $0.02 \pm 0.01$ \\
\hline
\end{tabular}

Min, minimum value; Max, maximum value; SD, standard error; ND, not detected.

\subsection{Beta Diversity}

In terms of beta diversity at the OTU level, unweighted and weighted unifrac PCoA showed that there were obvious varieties between individuals for the fungal community composition. Separation of dorsal colon from caecum occurred along the first axis (PC1) of the unweighted PCoA, but no separation of caecum from ventral colon was seen (Figure 7a). No obvious separation of the samples by hindgut region was observed in the weighted PCoA (Figure 7b).

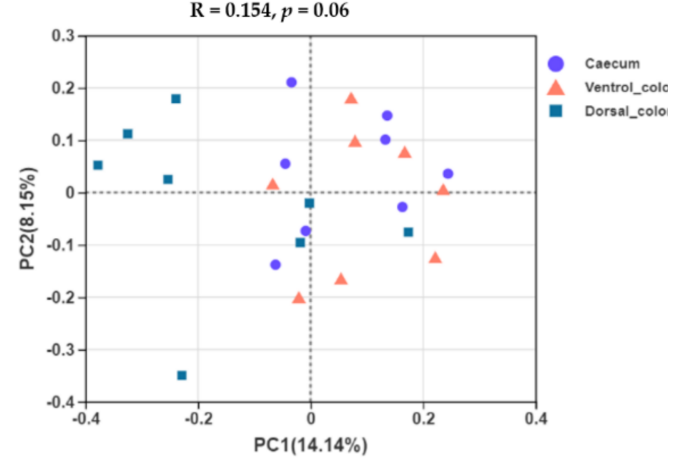

(a)

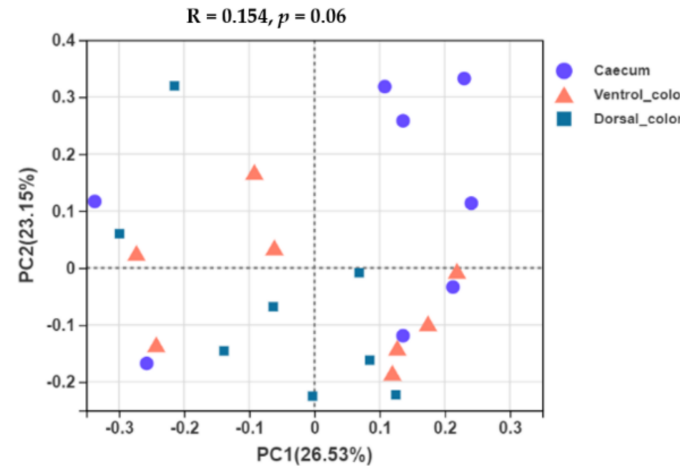

(b)

Figure 7. Unweighted (a) and weighted (b) unifrac based principal coordinates analysis (PCoA) of the fungal community composition of the different hindgut regions at the OTU level. Analysis used $\log _{10}$ transformed data, and the percentage values given on each axis indicate the amount of total variation represented. PC1, first axis; PC2, second axis. 


\subsection{Prediction of Fungal Enzymatic Activity by PICRUSt (Phylogenetic Investigation of Communities by Reconstruction of Unobserved States)}

The relative abundance of enzymes related to plant cell wall degradation were predicted by PICRUSt (Figure 8). These enzymes mainly included glucan 1,4-alpha-glucosidase beta-glucosidase, glucan 1,3-beta-glucosidase, alpha-glucosidase, glucan endo-1,3-alphaglucosidase, beta-glucuronidase, glucan 1,3-alpha-glucosidase, cellulase, pectinesterase, endo-1,4-beta-xylanase, arabinan endo-1,5-alpha-L-arabinosidase, feruloyl esterase, xylan 1,4-beta-xylosidase, glucan endo-1,3-beta-D-glucosidase, endo-1,3(4)-beta-glucanase, cellulose 1,4-beta-cellobiosidase (non-reducing end), and oligo-1,6-glucosidase, acetylxylan esterase. The relative abundances of these enzymes were lower in caecum than in both ventral and dorsal colon.
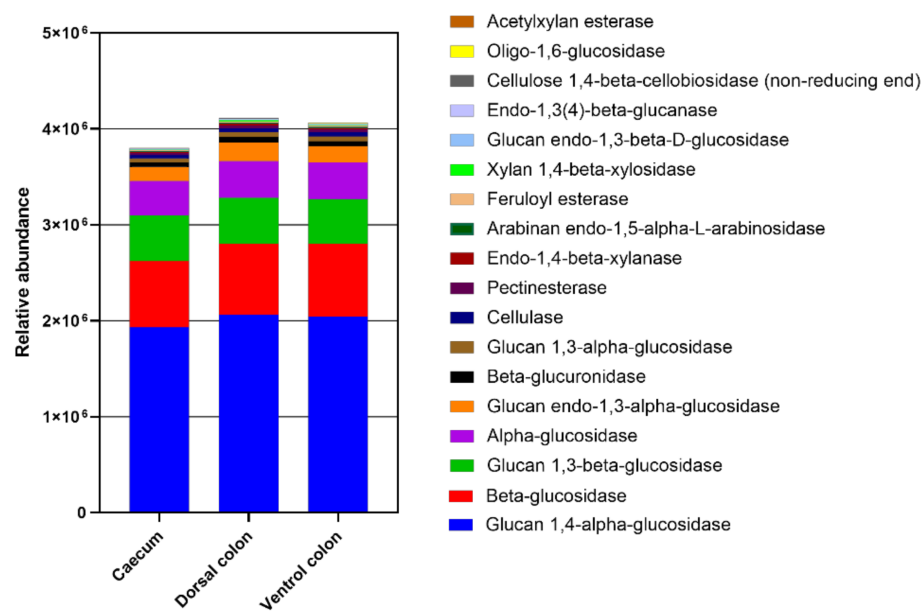

Figure 8. The abundance of enzymes related to plant cell wall degradation predicted by phylogenetic investigation of communities by reconstruction of unobserved states (PICRUSt). PICRUSt is a bioinformatics software package designed to predict metagenome functional content from marker gene (e.g., ITS) surveys.

\section{Discussion}

\subsection{Fibrolytic Enzyme Profiles}

The donkey hindgut is well developed and comprised of the several chambers, mainly including caecum, ventral colon, dorsal colon, and rectum [21]. In the previous study, Miyaji et al. reported that the cumulative disappearance of dietary fiber from the caecum to the rectum attained $90 \%$ of total tract digestion, indicating that the cecum and colon are the main regions for plant fiber fermentation in equines [22]. As equines themselves lack fibrolytic enzymes, the hindgut microorganisms are key to the ability of equines to hydrolyze dietary fiber [2]. Both the bacteria and the fungi can degrade dietary fiber efficiently via secreting a range of fibrolytic enzymes, such as free enzymes and cellulase multienzyme complexes [23]. Until now, there was a critical lack of knowledge regarding the fibrolytic activity among donkey hindgut. In the present study, the main-chain degrading enzymes (CMCase, AVI and XLY) and the side-chain degrading enzymes (AE) were thoroughly determined in donkey hindgut, and the activities were higher in colon in comparison with caecum. The fibrolytic activity may be related to the physical structure and chemical composition of the fibrous feeds [24]. With the incessant passage of the liquid and solid digesta in donkey hindgut, the structures of carbohydrate sources were changed and can regulate fibrolytic enzyme production. The increased lignin content and crosslinking between lignin polymers and polysaccharides in the colon digesta may promote the fibrolytic activity [25], especially the AE activity. Esterase is usually required to hydrolyze the cross-linkages in plant cell wall through phenolic compounds [4]. 


\subsection{Fungal Community in Donkey Caecum and Colon}

In recent years, study on the equine hindgut microbiota has increasingly improved with the advances in high throughput sequencing technology $[3,4,26]$. PCR amplification of universal primers for conserved regions within the rRNA genes, followed by DNA sequencing of the internal transcribed spacer (ITS), is widely used in fungal identification studies [27]. In the present study, we focused on the taxonomic affiliation of hindgut fungi by covering the ITS region with fungal wide primers (ITS1F ITS2R) $[18,19]$. The results provide new information about donkey caecum and colon fungi communities. At phylum level, Ascomycota, Basidiomycota, Neocallimastigomycota, and unclassified fungi were the predominant fungi within donkey caecum and colon. The present result is in agreement with the result of Zhang et al. [7] and Han et al. [8] in ruminant animals. Until now, knowledge of hindgut fungi and their influence on the equine animal remains limited. Using high-throughput sequencing to study the effect of hindgut region on fungal diversity and composition in donkeys has not been described. Edwards et al. [2] determined the fungal composition of fecal samples from donkey (Equus africanus asinus), however, they just target the phylum Neocallimstigomycta. These inconsistencies on phylum level might be due to differences in primers used for sequencing. Nowadays, both the ITS region and LSU (28S rRNA gene; D1/D2) region have been recommended as the universal DNA barcode marker for fungi [11]. However, a sufficient reference database is lacking for LSU taxonomic marker genes so far [5]. It is likely that ITS will continue to be commonly used in the near future as the primary barcode to evaluate fungal diversity and community composition.

In the current study, although the Ascomycota and Basidiomycota may be the dominant fungus in donkey caecum and colon, donkey hindgut region was just associated with differences in phylum Neocallimstigomycta. The relative abundance of Neocallimastigomycota in donkey caecum was significantly lower than that in the ventral and dorsal colon. In ruminants, previous studies reported that the Neocallimastigomycota was the most predominant fungal phylum [28,29], and they were important in the degradation of fibrous plant materials in ruminants $[7,30]$. The Neocallimastigomycota was presumed to be an effective plant fiber degrader and is well-known for its powerful hydrolytic properties and multi-functional fibrolytic enzymes [30]. Therefore, the larger abundant of Neocallimastigomycota in colon digesta may enable a donkey to degrade plant fiber more effectively compared to caecum.

At genus level, Aspergillus, Wallemia, Phanerochaete, Fusarium, and Penicillium were the predominant genera in current study. The genus Aspergillus is a group of filamentous fungi with a large number of species, and they have an effective effect on plant cell wall breakdown by producing a wide range of fibrolytic enzymes [31]. The enzymes act not only on the main chain of plant cell wall polysaccharides, but also act on the substituents or the side chains like the linkages between a main-chain residue and a substituent [30]. The Wallemia is another xerophilic filamentous fungi with the ability to secret a series of glycosidases [32]. The glycosidase was able to degrade both $\alpha$ - and $\beta$ - linked glycosidic bonds among di- and polysaccharides [33]. In addition, the Phanerochaete, Fusarium, and Penicillium were also reported to have the ability to produce various highly active enzymes, including cellulase, xylanase, and esterase [34-36]. From this study, the findings indicate that fungal community composition differs between different hindgut regions. The abundance of Fusarium and Trichosporon is greater in caecum than in dorsal colon. In contrast, the unclassified_Dipodascaceae and Cyllamyces in donkey colon was greater than in caecum. Nevertheless, as these genera were rarely found in herbivores, their metabolic and functional significance in donkey caecum-colon ecosystem need further investigation.

As strictly anaerobic fungi, Neocallimastigomycota have been most extensively studied in ruminants, but their information within equine animals is sparse. Recently, Edwards et al. observed that genus Caecomyces was the most predominant in the anaerobic fungal community of donkey fecal samples [2]. This is in agreement with the present study. The genus Caecomyces, belonging to Neocallimastigomycota phylum, was also detected as the dominant anaerobic fungi in donkey hindgut. In addition, the anaerobic fungi genera including Neocallimastix, Piromyces, Buwchfawromyces, Unclassified_f_Neocallimastigaceae 
and unclassified_c_Neocallimastigomycetes were also obtained from the donkey caecum and colon. The genus Neocallimastix, Piromyces, and Buwchfawromyces were cultured from equines [2,6]. However, Edwards et al. (2020) also observed the uncultivated genus SK3 and KF1 in donkey faeces, which was different from our findings [2]. The different donkey breeds and sample types may cause the distinct fungal compositions [37]. In addition, faecal samples did not accurately reflect the fungal community composition over the rest of the regions of the donkey hindgut [38]. For ruminant animals, Kittelmann et al. [39] noted that Neocallimastix, Piromyces, Orpinomyces, BlackRhino, Caecomyces, and Cyllamyces were the predominant anaerobic fungal genera in the rumen. This is perhaps not surprising considering the fundamental differences between hindgut herbivores and ruminants in terms of the main gut site where plant fiber degradation primarily occurs [5]. However, the genus Neocallimastix, Piromyces, and Cyllamyces were found to be shared in donkey hindgut and rumen. A previous study has shown that Neocallimastix is efficient in straw silage cell wall digestion [40], and it showed high polysaccharide hydrolase and esterase activity [41]. Piromyces could regulate the secretion of lignin-regulating enzymes via the fungal pathway and is capable of producing effective enzyme complex [42]. Screening of the microbial CAZyme transcripts indicated that the anaerobic fungi Neocallimastigaceae produced the largest share of cellulase transcripts [43]. The relative abundance of Neocallimastix, Piromyces, Buwchfawromyces, Unclassified_f_Neocallimastigaceae, and unclassified_c_Neocallimastigomycetes were numerically greater in colon than in caecum, which may enable the colon microorganisms to producing more fibrolytic enzymes than caecum microorganisms. Further study will be required to confirm the present observations.

\subsection{Prediction of Fungal Enzymatic Abundance by PICRUSt}

Due to the broad array of powerful plant degrading enzymes and the combined invasive growth, the fungi are key for herbivores to degrade plant cell walls [2]. There is a rare study performed on the fungal enzymes within equine animals. PICRUSt is a bioinformatics software package designed to predict metagenome functional content from marker gene (e.g., ITS). In the present study, the relative abundance of carbohydrate-active enzymes related to the degradation of cellulose, hemicellulose, pectin, and lignin were predicted. Interestingly, the relative abundance of these fibrolytic enzymes (glucan 1,4-alpha-glucosidase beta-glucosidase, glucan 1,3-beta-glucosidase, al-pha-glucosidase, glucan endo-1,3-alphaglucosidase, beta-glucuronidase, glucan 1,3-alpha-glucosidase, cellulase, pectinesterase, endo-1,4-beta-xylanase, arabinan endo-1,5-alpha-L-arabinosidase, feruloyl esterase, xylan 1,4-beta-xylosidase, glucan endo-1,3-beta-D-glucosidase, endo-1,3(4)-beta-glucanase, cellulose 1,4-beta-cellobiosidase (non-reducing end), oligo-1,6-glucosidase, and acetylxylan esterase) were greater in colon than in caecum. Considering that the relative abundance of Neocallimastigomycota was significantly greater in donkey colon than in colon, the higher enzyme content for plant cell wall breakdown in donkey colon may be related to their greater Neocallimastigomycota abundance. This result might consequently lead to the more efficient activity in dietary fiber digestion within donkey colon, relative to caecum.

\subsection{Welfare of the Animals}

With the unique cecum-colon ecosystem, donkeys obtain nutrients and energy from high fiber diets through microbial fermentation [44]. Information regarding the basic structure of the microbial flora in donkey hindgut is of vital importance in determining which components of the microflora respond to dietary variations [44]. The anaerobic fungi community and relative enzymes reported in the present study may provide a concrete reference for understanding donkey nutritional needs and health implications. It was because the donkey hindgut inhabited a large number of anaerobic bacteria and fungi that made donkeys highly efficient at digesting poor nutritional quality fiber [45]. Forages were the basis of donkey's diet, and the concentrated meals were discouraged from use [46]. Donkeys rarely require energy-rich concentrate, such as cereal grains, sweet feeds, or highly crushed feed [45]. Due to the high concentration of soluble carbohydrates 
in cereal grain diets, the feeding of such concentrates for donkey is poorly tolerated. When concentrates were given over and above the capacity of the foregut to digest them, the increased soluble carbohydrates will enter the caecum and colon [44,47]. This was implicated in the development of health problems, such as obesity, hyperlipemia, equine acidosis, and colic $[45,48]$. In addition, feeding more of less energy-rich feeds may also allow larger meals, giving the animals longer eating times. Therefore, healthy donkeys in practical feeding should focus on optimizing fiber utilization and providing greater proportions of highly fibrous feedstuffs, such as crop straw, grass hay, and haylage being fed as required, according to body condition and life stage [45].

\section{Conclusions}

The fibrolytic enzyme profiles within different donkey hindgut regions were firstly measured in the present study. Activities of carboxymethyl cellulase, avicelase, xylanase, and acetyl esterase were greater in donkey dorsal colon than in caecum, indicating that the colon microorganisms may be more efficient in producing fibrolytic enzymes compared to caecum microbes. The fungal community composition along donkey hindgut segments using Illumina MiSeq sequencing by covering the ITS region were determined. The predominant fungi at phylum level in donkey caecum and colon were Ascomycota (66.8-74.4\%), Basidiomycota (21.6-30.9\%), Neocallimastigomycota (0.9-3.3\%) and unclassified_fungi (1.01.3\%). The Aspergillus, Wallemia, Phanerochaete, Fusarium, and Penicillium were detected as the dominant genera in donkey caecum and colon, but their metabolic and functional significance in donkey caecum-colon ecosystem need further investigation. The hindgut region of donkey was associated with differences in fungal community composition. The relative abundance of Neocallimastigomycota in dorsal colon was greater in donkey colon than in caecum, and the genera Cyllamyces, Neocallimastix, Piromyces, Buwchfawromyces, unclassified_f_Neocallimastigaceae, and unclassified_c_Neocallimastigomycetes belong to Neocallimastigomycota phylum were also numerically greater in colon than in caecum. This result indicated that the high proportion of strictly anaerobic fungi in colon may enable a donkey to degrade plant fiber more effectively, compared to caecum. Moreover, the relative abundance of enzymes related to plant cell wall degradation were predicted by PICRUSt, and the relative abundances of these enzymes were also lower in caecum than in both ventral colon and dorsal colon. The findings in the current study could therefore contribute to the further understanding of the fungal taxa and their plant fiber degradation mechanisms in donkey hindgut ecosystem.

Author Contributions: Conceptualization, Z.Z. and C.W.; methodology, Z.Z. and C.W.; software, Z.Z.; formal analysis, Z.Z.; investigation, Z.Z., Y.W. and B.H.; data curation, Z.Z.; writing-original draft preparation, Z.Z.; writing—review and editing, Z.Z., M.Z. and C.W.; project administration, C.W.; funding acquisition, C.W. All authors have read and agreed to the published version of the manuscript.

Funding: This research was funded by the Well-bred Program of Shandong Province (grant no. 2017LZGC020), Taishan Leading Industry Talents Agricultural Science of Shandong Province (grant no. LJNY201713), Shandong Province Modern Agricultural Technology System Donkey Industrial Innovation Team (grant no. SDAIT-27), The Open Project of Liaocheng Universtiy Animal Husbandry Discipline (grant no. 319312101-14), The Open Project of Shandong Collaborative Innovation Center for Donkey Industry Technology (grant no. 3193308), Research on Donkey Pregnancy Improvement (grant no. K20LC0901), and Liaocheng University scientific research fund (grant no. 318052025).

Institutional Review Board Statement: The study was conducted according to the guidelines of the Declaration of Helsinki, and approved by the Institutional Animal Care Committee at Liaocheng University (Permit No. DFG21010103-1).

Informed Consent Statement: Not applicable.

Data Availability Statement: The data that support the findings of this study are available from the authors.

Conflicts of Interest: The authors declare no conflict of interest. 


\section{References}

1. Murrua, F.; Fliegerova, K.; Mura, E.; Mrázek, J.; Kopečný, J.; Monielloa, G. A comparison of methanogens of different regions of the equine hindgut. Anaerobe 2018, 54, 104-110. [CrossRef]

2. Edwards, J.E.; Schennink, A.; Burden, F.; Long, S.; Smidt, H. Domesticated equine species and their derived hybrids differ in their fecal microbiota. Anim. Microbiome 2020, 2, 8. [CrossRef]

3. Liu, G.Q.; Bou, G.; Su, S.F.; Xing, J.Y.; Qu, H.L.; Zhang, X.Z.; Wang, X.S.; Zhao, Y.P.; Dugarjaviin, M. Microbial diversity within the digestive tract contents of Dezhou donkeys. PLOS ONE 2019, 14, e0226186. [CrossRef]

4. Liu, H.; Zhao, X.; Han, X.; Xu, S.; Chen, X. Comparative study of gut microbiota in tibetan wild asses (equus kiang) and domestic donkeys (Equus asinus) on the qinghai-tibet plateau. PeerJ 2020, 8, e9032. [CrossRef] [PubMed]

5. Hess, M.; Paul, S.S.; Puniya, A.K.; van der Giezen, M.; Shaw, C.; Edwards, J.E.; Fliegerová, K. Anaerobic fungi: Past, present, and future. Front. Microbiol. 2020, 11, 584893. [CrossRef]

6. Edwards, J.E.; Shetty, S.A.; Berg, P.V.D.; Burden, F.; Smidt, H. Multi-kingdom characterization of the core equine fecal microbiota based on multiple equine (sub)species. Anim. Microbiome 2020, 2, 6. [CrossRef]

7. Zhang, J.; Shi, H.; Wang, Y.; Li, S.; Cao, Z.; Ji, S.; He, Y.; Zhang, H. Effect of dietary forage to concentrate ratios on dynamic profile changes and interactions of ruminal microbiota and metabolites in Holstein heifers. Front. Microbiol. 2017, 9, 2206-2223. [CrossRef] [PubMed]

8. Han, X.; Li, B.; Wang, X.; Chen, Y.; Yang, Y. Effect of dietary concentrate to forage ratios on ruminal bacterial and anaerobic fungal populations of cashmere goats. Anaerobe 2019, 59, 118-125. [CrossRef]

9. $\quad$ Edwards, J.E.; Forster, R.J.; Callaghan, T.M.; Dollhofer, V.; Dagar, S.S.; Cheng, Y.; Chang, J.; Kittelmann, S.; Fliegerova, K.; Puniya, A.K.; et al. PCR and Omics Based Techniques to Study the Diversity, Ecology and Biology of Anaerobic Fungi: Insights, Challenges and Opportunities. Front. Microbiol. 2017, 8, 1657. [CrossRef]

10. Vinzelj, J.; Joshi, A.; Insam, H.; Podmirseg, S.M. Employing anaerobic fungi in biogas production: Challenges \& opportunities Bioresour. Technol. 2020, 300, 122687.

11. Fliegerova, K.O.; Podmirseg, S.M.; Vinzelj, J.; Grilli, D.J.; Moniello, G. The effect of a high-grain diet on the rumen microbiome of goats with a special focus on anaerobic fungi. Microorganisms 2021, 9, 157. [CrossRef] [PubMed]

12. Edwards, J.E.; Hermes, G.D.A.; Kittelmann, S.; Nijsse, B.; Smidt, H. Assessment of the accuracy of high-throughput sequencing of the ITS1 region of Neocallimastigomycota for community composition analysis. Front. Microbiol. 2019, 10, 2370. [CrossRef] [PubMed]

13. Santos, A.S.; Rodrigues, M.A.M.; Bessa, R.J.B.; Ferreira, L.M.; Martin-Rosset, W. Understanding the equine cecum-colon ecosystem: Current knowledge and future perspectives. Animal 2011, 5, 48-56. [CrossRef] [PubMed]

14. Gordon, G.L.R.; Phillips, M.W. The role of anaerobic gut fungi in ruminants. Nutr. Res. Rev. 1998, 11, 133-168. [CrossRef]

15. Agina, O.A. Haematology and Clinical Biochemistry Findings Associated with Equine Diseases-A Review. Not. Sci. Biol. 2017, 9, 1-21. [CrossRef]

16. Yue, Q.; Yang, H.J.; Cao, Y.C.; Zhang, D.F.; Jiang, Y.H.; Wang, J.Q. Feruloyl and acetyl esterase production of an anaerobic rumen fungus neocallimastix sp. yq2 effected by glucose and soluble nitrogen supplementations and its potential in the hydrolysis of fibrous feedstuffs. Anim. Feed. Sci. Technol. 2009, 153, 263-277. [CrossRef]

17. Colombatto, D.; Morgavi, D.P.; Furtado, A.F. Screening of exogenous enzymes for ruminant diets: Relationship between biochemical characteristics and in vitro ruminal degradation. J. Anim. Sci. 2003, 81, 2628-2638. [CrossRef]

18. Hristov, A.N.; Lee, C.; Cassidy, T.; Heyler, K.; Tekippe, J.A.; Varga, G.A.; Corl, B.; Brandt, R.C. Effect of Origanum vulgare L. leaves on rumen fermentation, production, and milk fatty acid composition in lactating dairy cows. J. Dairy Sci. 2013, 96, 1189-1202. [CrossRef]

19. Man, B.; Wang, H.; Yun, Y.; Xiang, X.; Wang, R.; Duan, Y.; Cheng, X. Diversity of fungal communities in Heshang Cave of Central China revealed by Mycobiome-Sequencing. Front. Microbiol. 2018, 16, 1400-1414. [CrossRef]

20. Wang, K.; Nan, X.; Chu, K.; Tong, J.; Yang, L.; Zheng, S.; Zhao, G.; Jiang, L.; Xiong, B. Shifts of hydrogen metabolism from methanogenesis to propionate production in response to replacement of forage fiber with non-forage fiber sources in diets in vitro. Front. Microbiol. 2018, 1, 9. [CrossRef]

21. Varloud, M.; de Fombelle, A.; Goachet, A.G.; Drogoul, C.; Julliand, V. Partial and total apparent digestibility of dietary carbohydrates in horses as affected by the diet. Anim. Sci. 2004, 79, 61-72. [CrossRef]

22. Miyaji, M.; Ueda, K.; Kobayashi, Y.; Hata, H.; Kondo, S. Fiber digestion in various segments of the hindgut of horses fed grass hay or silage. Anim. Sci. J. 2008, 79, 339-346. [CrossRef]

23. Cheng, Y.; Shi, Q.; Sun, R.; Liang, D.; Li, Y.; Li, Y.; Jin, W.; Zhu, W. The biotechnological potential of anaerobic fungi on fiber degradation and methane production. World J. Microbiol. Biotechnol. 2018, 34, 155-162. [CrossRef] [PubMed]

24. Du, L.; Yu, P.; Rossnagel, B.G.; Christensen, D.A.; Mckinnon, J.J. Physicochemical characteristics, hydroxycinnamic acids (ferulic acid, p-coumaric acid) and their ratio, and in situ biodegradability: Comparison of genotypic differences among six barley varieties. J. Agric. Food Chem. 2009, 57, 4777-4783. [CrossRef] [PubMed]

25. Yang, H.J.; Yue, Q.; Cao, Y.C.; Zhang, D.F.; Wang, J.Q. Effects of crude feruloyl and acetyl esterase solutions of neocallimastix sp. yq1 and anaeromyces sp. yq3 isolated from holstein steers on hydrolysis of chinese wildrye grass hay, wheat bran, maize bran, wheat straw and corn stalks. Anim. Feed. Sci. Technol. 2009, 154, 218-227. [CrossRef] 
26. Julliand, V.; Grimm, P. Horse species symposium: The microbiome of the horse hindgut: History and current knowledge. J. Anim. Sci. 2016, 94, 2262-2274. [CrossRef]

27. Pryce, T.M.; Palladino, S.; Price, D.M.; Gardam, D.J.; Campbell, P.B.; Christiansen, K.J.; Murray, R.J. Rapid identification of fungal pathogens in BacT/ALERT, BACTEC, and BBL MGIT media using polymerase chain reaction and DNA sequencing of the internal transcribed spacer regions. Diagn. Microbiol. Infect. Disease 2006, 54, 289-297. [CrossRef]

28. Koetschan, C.; Kittelmann, S.; Lu, J.; Al-Halbouni, D.; Jarvis, G.N.; Muller, T.; Wolf, M.; Janssen, P.H. Internal transcribed spacer 1 secondary structure analysis reveals a common core throughout the anaerobic fungi (Neocallimastigomycota). PLoS ONE 2014, 9, e91928. [CrossRef]

29. Mao, S.Y.; Huo, W.J.; Zhu, W.Y. Microbiome-metabolome analysis reveals unhealthy alterations in the composition and metabolism of ruminal microbiota with increasing dietary grain in a goat model. Environ. Microbiol. 2016, 18, 525-541. [CrossRef]

30. Gruninger, R.J.; Puniya, A.K.; Callaghan, T.M.; Edwards, J.E.; Youssef, N.; Dagar, S.S.; Fliegerova, K.; Griffith, G.W.; Forster, R.; Tsang, A.; et al. Anaerobic fungi (phylum Neocallimastigomycota): Advances in understanding their taxonomy, life cycle, ecology, role and biotechnological potential. FEMS Microbiol. Ecol. 2014, 90, 1-17. [CrossRef]

31. Ralet, M.C.; Faulds, C.B.; Williamson, G.; Thibault, J.F. Degradation of feruloylated oligosaccharides from sugar beet pulp and wheat bran by ferulic acid esterases from Aspergillus niger. Carbohydr. Res. 1994, 263, 257-269. [CrossRef]

32. Miller, T.A. Purification and Characterisation of a Secreted Glycosidase, from the Extreme Xerophile Wallemia ichthyophaga. Master's Thesis, Massey University, Palmerston North, Wellington, New Zealand, 2014.

33. Hanafy, R.A.; Elshahed, M.S.; Youssef, N.H. Feramyces austinii, gen. nov., sp. nov., an anaerobic gut fungus from rumen and fecal samples of wild barbary sheep and fallow deer. Mycologia 2018, 110, 513-525. [CrossRef]

34. Saparrat, M.C.N.; Martínez, M.J.; Tournier, H.A.; Cabello, M.N.; Arambarri, A.M. Production of ligninolytic enzymes by Fusarium solani strains isolated from different substrata. World J. Microbiol. Biotechnol. 2000, 16, 799-803. [CrossRef]

35. Moredo, N.; Lorenzo, M.; Domínguez, A.; Moldes, D.; Cameselle, C.; Sanroman, A. Enhanced ligninolytic enzyme production and degrading capability of Phanerochaete chrysosporium and Trametes versicolor. World J. Microbiol. Biotechnol. 2003, 19, 665-669. [CrossRef]

36. Narasimha, G.; Sridevi, A.; Devi, P.S. Production of ligninolytic enzymes from Penicillium sp. and its efficiency to decolourise textile dyes. Open Biotechnol. J. 2018, 12, 112-122.

37. Dougal, K.; Harris, P.A.; Edwards, A.; Pachebat, J.A.; Blackmore, T.M.; Worgan, H.J.; Newbold, C.J. A comparison of the microbiome and the metabolome of different regions of the equine hindgut. FEMS Microbiol. Ecol. 2012, 82, 642-652. [CrossRef] [PubMed]

38. Tap, J.; Mondot, S.; Levenez, F. Towards the human intestinal microbiota phylogenetic core. Environ. Microbiol. 2009, 11, 2574-2584. [CrossRef]

39. Kittelmann, S.; Seedorf, H.; Walters, W.A.; Clemente, J.C.; Knight, R.; Gordon, J.I.; Janssen, P.H. Simultaneous amplicon sequencing to explore co-occurrence patterns of bacterial, archaeal and eukaryotic microorganisms in rumen microbial communities. PLoS ONE 2013, 8, e47879.

40. Lee, S.M.; Guan, L.L.; Eun, J.S.; Kim, C.H.; Lee, S.J.; Kim, E.T.; Lee, S.S. The effect of anaerobic fungal inoculation on the fermentation characteristics of rice straw silages. J. Appl. Microbiol. 2015, 118, 565-573. [CrossRef]

41. Wei, Y.Q.; Long, R.J.; Yang, H.; Yang, H.J.; Shen, X.H.; Shi, R.F.; Wang, Z.Y.; Du, J.G.; Qi, X.J.; Ye, Q.H. Fiber degradation potential of natural co-cultures of Neocallimastix frontalis and Methanobrevibacter ruminantium isolated from yaks (Bos grunniens) grazing on the Qinghai Tibetan Plateau. Anaerobe 2016, 39, 158-164. [CrossRef]

42. Hooker, C.A.; Hillman, E.T.; Overton, J.C.; Ortiz-Velez, A.; Schacht, M.; Hunnicutt, A.; Mosier, N.S.; Solomon, K.V. Hydrolysis of untreated lignocellulosic feedstock is independent of S-lignin composition in newly classified anaerobic fungal isolate, Piromyces sp. UH3-1. Biotechnol. Biofuels 2018, 11, 293-306. [CrossRef] [PubMed]

43. Söllinger, A.; Tveit, A.T.; Poulsen, M.; Noel, S.J.; Bengtsson, M.; Bernhardt, J.; Hellwing, A.L.F.; Lund, P.; Riedel, K.; Schleper, C.; et al. Holistic assessment of rumen microbiome dynamics through quantitative metatranscriptomics reveals multifunctional redundancy during key steps of anaerobic feed degradation. mSystems 2018, 3, e00038-18. [CrossRef] [PubMed]

44. Daly, K.; Stewart, C.S.; Flint, H.J.; Shirazi-Beechey, S.P. Bacterial diversity within the equine large intestine as revealed by molecular analysis of cloned 16s rrna genes. FEMS Microbiol. Ecol. 2001, 38, 141-151. [CrossRef]

45. Burden, F.; Thiemann, A. Donkeys are different. J. Equine Vet. Sci. 2015, 35, 376-382. [CrossRef]

46. Raspa, F.; Cavallarin, L.; Mclean, A.K.; Bergero, D.; Valle, E. A review of the appropriate nutrition welfare criteria of dairy donkeys: Nutritional requirements, farm management requirements and animal-based indicators. Animals 2019, 9, 315. [CrossRef]

47. Garner, H.E.; Hutcheson, D.P.; Cojman, J.R.; Hahn, A.W.; Salem, C. Lactic acidosis: A factor associated with equine laminitis. J. Anim. Sci. 1977, 45, 1037-1041. [CrossRef] [PubMed]

48. Martin-Rosset, W. Donkey nutrition and feeding: Nutrient requirements and recommended allowances-a review. J. Equine Vet. Sci. 2018, 65, 75-85. [CrossRef] 